\title{
Características clínico-epidemiológicas y percepción de los padres sobre el estreñimiento funcional en la infancia en una población hospitalaria
}

\author{
Clinico-epidemiological characteristics and parental \\ perception of functional constipation in childhood in a \\ hospital population
}

\author{
Daniela Mallada ${ }^{1}$, José Lacarrubba²® ${ }^{2}$ Lidia Garcete ${ }^{3} \bullet$ \\ ${ }^{1}$ Hospital Materno Infantil San Pablo. Servicio de Urgencias Pediátricas. Asunción, Paraguay. \\ ${ }^{2}$ Universidad Nacional de Asunción. Facultad de Ciencias Médicas. Hospital de Clínicas. Departamento \\ de Neonatología. San Lorenzo, Paraguay. \\ ${ }^{3}$ Universidad Nacional de Asunción. Facultad de Ciencias Médicas. Cátedra y Servicio de Pediatría. San \\ Lorenzo, Paraguay.
}

\section{RESUMEN}

Introducción: El estreñimiento funcional (EF) en niños es un problema común, constituyendo un importante problema de salud. Objetivo: Caracterización clínicoepidemiológica y percepción de padres y/o cuidadores de niños de una consulta pediátrica sobre el EF en la infancia. Materiales y Métodos: Estudio observacional descriptivo de corte transverso, utilizando la entrevista con encuesta semiestructurada a cuidadores de niños de 2 a 10 años de una Unidad Pediátrica Ambulatoria. Resultados: sobre 374 encuestados 251(67\%) niños estaba al cuidado de sus padres y provenían del departamento central. La frecuencia de $\mathrm{EF}$ fue de 4,8\% (4.4\% en preescolares y 5,1\% en escolares). En escolares, predominó en varones. En niños con EF se refirió defecación dolorosa en $66,6 \%$, antecedente familiar de estreñimiento en $61 \%$, ingesta excesiva de lácteos en 39\% y $17 \%$ no consumía ninguna fruta o vegetal. El $55,5 \%$ de niños inició entrenamiento esfinteriano antes de los 24 meses. Se observó $11 \%$ de cuidadores sin escolaridad. Desde la percepción de los encuestados se consideró estreñimiento: esfuerzo defecatorio $(27 \%)$, ausencia de defecación diaria $(19,7 \%)$, deposiciones duras y dolorosas $(34,4 \%$ ) y necesidad de uso de laxantes (18,9\%). 367/374 (98.1\%) conocía los hábitos intestinales de los niños a su cargo en frecuencia, características y síntomas. Refirieron utilización de supositorios, laxantes y enemas en $100 \%$ en EF. Conclusiones: La frecuencia del EF fue inferior al

\section{ABSTRACT}

Introduction: Functional constipation (FC) in children is a common problem, constituting an important health problem. Objective: To determine the clinicalepidemiological characteristics and the perception of parents and / or caregivers of children who consulted because of FC in childhood. Materials and Methods: This was a cross-sectional descriptive and observational study, using interviews with a semi-structured survey with caregivers of children aged 2 to 10 years in a Pediatric Outpatient Unit. Results: out of 374 respondents, 251 (67\%) children were in the care of their parents and came from the Central Department. The frequency of FC was $4.8 \%$ (4.4\% in preschool and $5.1 \%$ in schoolchildren). In schoolchildren, it predominated in boys. In children with FC, painful defecation was reported in $66.6 \%$, a family history of constipation in $61 \%$, excessive dairy intake in $39 \%$, and $17 \%$ did not consume any fruit or vegetable. $55.5 \%$ of children began sphincter training before 24 months. $11 \%$ of caregivers were without schooling. From the respondents' perspective, constipation was considered: defecation effort (27\%), absence of daily defecation (19.7\%), hard and painful stools (34.4\%) and need to use laxatives (18.9\%). 367/374 (98.1\%) knew the intestinal habits of the children in their care in terms of frequency, characteristics and symptoms. They reported the use of suppositories, laxatives and enemas in 100\% in FC. Conclusions: The frequency of FC was lower than the reported world

Correspondencia: Daniela Mallada. Correo: malladamdaniela@gmail.com

Recibido: 23/10/2020 Aceptado:16/02/2021

Conflictos de interés: Los autores declaran no presentar conflicto de interés.

DOI: https://doi.org/10.31698/ped.48012021009

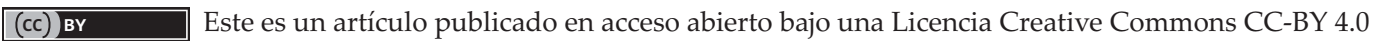


promedio mundial reportado de $12 \%$, con predominio en varones en edad escolar. Se constató antecedente familiar de estreñimiento, conceptualización poco clara sobre EF, prácticas inapropiadas sobre prevención y manejo del EF.

Palabras clave: Estreñimiento, incontinencia fecal, niños. average of $12 \%$, with a predominance in school-age males. There was a family history of constipation, unclear conceptualization of $\mathrm{FC}$, inappropriate practices in prevention and management of FC.

Key words: Constipation, fecal incontinence, children.

\section{INTRODUCCIÓN}

El estreñimiento funcional (EF) asocia trastornos en los hábitos intestinales con disminución de frecuencia, heces duras, voluminosas, esfuerzo defecatorio, dolor, y alteraciones conductuales como negativa a defecar, posturas de evitación y pérdidas fecales, constituyendo un problema complejo que afecta a niños de todo el mundo, edades y $\operatorname{sexo}^{(1)}$. Su prevalencia mundial oscila entre $0,7-29 \%$, con una media del $12 \%{ }^{(2)}$ y representa 3\% de consultas al pediatra, $25 \%$ al gastroenterólogo ${ }^{(3)}$ y 3 a $16 \%$ a urgencias ${ }^{(4)}$.

El $95 \%$ de casos de estreñimiento obedece a un trastorno funcional. La defecación dolorosa es el factor desencadenante principal de la retención fecal, generando un círculo vicioso ${ }^{(5)}$.

La etiología y fisiopatología son complejas. Puede coincidir con el inicio de la alimentación complementaria, del entrenamiento esfinteriano o la escolarización. Un entrenamiento esfinteriano coercitivo puede favorecerlo. La etapa escolar es crítica, por la adaptación a horarios y sanitarios públicos ${ }^{(2)}$. Entre los factores asociados se mencionan: ingesta pobre de fibra dietética y líquidos, exceso de lácteos, edad precoz de retirada del pañal, sedentarismo, historia familiar positiva, cambios en la rutina diaria, intimidación escolar, trastornos emocionales y eventos estresantes $^{(6)}$. Como factores ambientales se citan bajo nivel educativo y estructura familiar.

El diagnóstico es clínico, utilizando los criterios del consenso de Roma IV, basado en una historia y exploración física compatibles ${ }^{(6)}$.

La información de los padres es clave. Se debe investigar momento de expulsión del primer meconio, edad al inicio de síntomas, características de las heces, dolor y sangrado rectal, historia dietética, pérdida de peso, síntomas urinarios coexistentes, uso de medicamentos que producen estreñimiento, supositorios, laxantes o enemas y comorbilidad psicosocial $^{(7)}$, para el diagnóstico diferencial, que incluye enfermedad de Hirschsprung, celiaquía, anomalías anatómicas, medulares, metabólicas y neuro entéricas ${ }^{(8)}$. Ante sospecha de anomalías serán necesarios: tacto rectal y exámenes complementarios ${ }^{(7)}$.

El manejo es complejo y la intervención precoz esencial, incluyendo ejercicio, dieta y modificación conductual. Si hay impactación fecal es necesario desimpactar prefiriéndose los laxantes osmóticos orales, no absorbibles ${ }^{(3)}$, reservando los enemas para situaciones de fracaso. Debe asegurarse la evacuación regular en el seguimiento ${ }^{(9)}$.

Considerando su relevancia, se establecieron como objetivos: Determinar las características clínicoepidemiológicas del EF y la interpretación o significado que otorgan padres y/o cuidadores de niños de una consulta pediátrica a los signos y síntomas del EF en la infancia.

\section{MATERIALES Y MÉTODOS}

Estudio observacional descriptivo de corte transverso, utilizando el método de entrevista con consentimiento informado. Se aplicó un cuestionario semi-estructurado a padres y/o cuidadores de niños de 2 a 11 años de edad que acudieron a la consulta pediátrica en la Unidad Pediátrica Ambulatoria (UPA) del Hospital de Clínicas de la Facultad de Ciencias MédicasUniversidad Nacional de Asunción, entre los meses de agosto, setiembre y octubre del año 2017. 
Fueron analizadas variables sociodemográficas y clínicas: edad, sexo, procedencia, hábitos intestinales, antecedentes familiares de estreñimiento, hábitos alimentarios y de entrenamiento esfinteriano, nivel de escolaridad de los cuidadores y tratamiento implementado en caso de presentar tanto EF como episodios de estreñimiento ocasional. Fueron igualmente analizadas las creencias, interpretación o significado que otorgan a los signos y síntomas relacionados al habito defecatorio de los niños.

Se definieron como preescolares los niños de 2 a 5 años $11 \mathrm{~m}$ y escolares de 6 a 10 años $11 \mathrm{~m}$, en base al sistema utilizado en el ciclo escolar nacional.

Para analizar la ingesta de fibra dietaria y de lácteos se utilizó el recordatorio de $24 \mathrm{hs}$ y se compararon las ingestas con las recomendaciones de las guías alimentarias del MINSAL de Chile para el preescolar y escolar ${ }^{(10)}$.

Para evaluar el tiempo considerado apropiado de entrenamiento esfinteriano se revisaron las pautas de desarrollo psicomotor del Curso de Actualización de Pediatría de AEPap $2016^{(11)}$.

Criterios de inclusión: Padres y/o cuidadores de niños de 2 a 10 años 11 meses de edad que acudieron a consulta de control de salud durante el periodo de tiempo de la recolección de datos y que aceptaron participar previa información sobre los propósitos de la investigación.

Criterios de exclusión: Padres y/o cuidadores de pacientes con patologías específicas que afecten el hábito intestinal (por ej, enfermedad celiaca, alergia a proteínas de leche de vaca, enfermedad de Hirschsprung, anomalías anorectales, alteraciones de la columna lumbosacra, metabólicas y neuro entéricas) y encuestas incompletas.

Para el diagnóstico de EF se utilizaron los criterios Roma IV, que fueron incluidos entre las variables investigadas en el cuestionario aplicado. Se consideró con EF a aquellos que cumplían los criterios Roma IV (lista)y sin EF a los que no cumplían con los criterios, pudiendo estos presentar episodios ocasionales de trastornos defecatorios.

\section{Criterios diagnósticos Roma IV.}

Debe incluir al menos dos de ellos durante al menos un mes:

1. Dos o menos deposiciones por semana.

2. Historia de retención fecal excesiva.

3. Historia de defecación dolorosa o heces duras.

4. Historia de deposiciones voluminosas.

5. Presencia de gran masa fecal en el recto.

En niños continentes fecales, pueden usarse los siguientes criterios adicionales:

6. Al menos un episodio semanal de incontinencia.

7. Historia de deposiciones voluminosas que obstruyen el sanitario

Tamaño de muestra: Se utilizo el valor estadístico de Z:1.9, se consideró una frecuencia de $9 \%$ de EF en población general, valor de D: $95 \%$, que define el intervalo de confianza del $95 \%$ y valor de p: 0,05, resultando $\mathrm{N}: 374$

Se utilizó el paquete estadístico SPSS versión 11.5. Para variables cualitativas se empleó el cálculo de frecuencias y porcentajes.

Se elaboro un protocolo que fue aprobado por la Dirección del Posgrado de Clínica Pediátrica de la FCM.UNA. Los informantes aceptaron participar previa información sobre los propósitos de la investigación, dentro de la confidencialidad y anonimato.

\section{RESULTADOS}

Fueron encuestados padres o cuidadores de 400 niños. Para el análisis estadístico se incluyeron 374 $(93,5 \%)$ encuestas y fueron excluidas $26(6,5 \%)$ ya que 12 se encontraban incompletas y 14 correspondían a pacientes con patología de base (ano imperforado, alergia a la proteína a la leche de vaca, enfermedad de Hirshprung).

Sobre la población de 374 niños, las edades oscilaron entre 2años y 10 años $11 \mathrm{~m}$, siendo similar la distribución de escolares 51,6\% (193/374) y 48,4\% (181/374) de preescolares. El 58,5\% (103/181) de los preescolares eran de sexo femenino y el $60,6 \%$ (120/193) de los escolares eran de sexo masculino. 
Cumplieron los criterios ROMA IV para EF 18/ 374 $(4.8 \%)$ niños. La frecuencia fue similar en preescolares y en escolares (Tabla 1 ).
En la tabla 2 se detalla la distribución por grupo etario y sexo de los participantes con y sin EF.

Tabla 1. Frecuencia de estreñimiento. En la población estudiada N=374

\begin{tabular}{llll}
\hline Grupo & $\begin{array}{l}\text { Con EF* } \\
\mathbf{n = 1 8}\end{array}$ & $\begin{array}{l}\text { Sin EF } \\
\mathbf{n = 3 5 6}\end{array}$ & Total \\
\hline Preescolar & $8(4,4 \%)$ & $173(48,6 \%)$ & $181(48,4 \%)$ \\
Escolar & $10(5,1 \%)$ & $183(51,4 \%)$ & $193(51,6 \%)$ \\
\hline Total & $\mathbf{1 8 ( 4 , 8 \% )}$ & $\mathbf{3 5 6}(\mathbf{9 5 , 2} \%)$ & $\mathbf{3 7 4}$ \\
\hline EF $=$ Estreñimiento funcional & &
\end{tabular}

Tabla 2. Distribución de la población con EF y sin EF según grupo de edad y sexo. $\mathrm{N}=374$

\begin{tabular}{llll}
\hline Grupo & $\begin{array}{l}\text { Con EF* } \\
\mathbf{n = 1 8}\end{array}$ & $\begin{array}{l}\text { Sin EF } \\
\mathbf{n}=\mathbf{3 5 6}\end{array}$ & Total \\
\hline $\begin{array}{l}\text { Preescolar } \\
\text { Femenino }\end{array}$ & $6(5,8 \%)$ & $97(94,2 \%)$ & 103 \\
Masculino & $2(2,6 \%)$ & $76(97,4 \%)$ & 78 \\
\hline Escolar & & $73(100 \%)$ & 73 \\
Femenino & 0 & $110(91,7 \%)$ & 120 \\
Masculino & $10(8,3 \%)$ & $\mathbf{3 5 6}(\mathbf{9 5 , 2} \%)$ & $\mathbf{3 7 4}$ \\
\hline Total & $\mathbf{1 8 ( 4 , 8 \% )}$ & & \\
\hline
\end{tabular}

$E F=$ Estreñimiento funcional

De los encuestados, 308(82\%) provenía del Departamento Central y de Asunción, en tanto que 66 (18\%) de los departamentos del interior del país, sin diferencias entre EF o sin EF.

Estaban bajo el cuidado de sus padres 12/18 (67\%) niños con EF y 239/356 (67\%) sin EF. Se encontraban bajo cuidado de terceros 33\% (6/18) niños EF y 32\% $(117 / 356) \sin \mathrm{EF}$.
Fue referido defecación dolorosa desde el inicio del EF en 12/18 (66,6\%) niños con EF y 82/356 (23\%) niños sin EF declararon episodios ocasionales de dolor.

Se observó antecedente familiar de estreñimiento en $11 / 18(61 \%)$ de los EF, siendo la madre la principal afectada (Tabla 3 )

Tabla 3. Antecedentes familiares de estreñimiento, según grupo con y sin Estreñimiento funcional. N=374

\begin{tabular}{lll}
\hline & $\begin{array}{l}\text { Con EF* } \\
\mathbf{n = 1 8}\end{array}$ & $\begin{array}{l}\text { Sin EF } \\
\mathbf{n}=\mathbf{3 5 6}\end{array}$ \\
\hline Madre & $7(63,7 \%)$ & $66(54 \%)$ \\
Abuelos & $3(27,3 \%)$ & $41(33,6 \%)$ \\
Padre & $1(9 \%)$ & $15(12,4 \%)$ \\
\hline Total & $\mathbf{1 1}(\mathbf{6 1 \% )}$ & $\mathbf{1 2 2 ( 3 5 , 5 \% )}$ \\
\hline EF = Estreñimiento funcional &
\end{tabular}

Sobre los hábitos de alimentación, consumían las porciones recomendadas de frutas solo $3 / 18(16,6 \%)$ de los EF y de los sin EF 100/356 (28\%), y la cantidad recomendada de vegetales $2 / 18(11.1 \%)$ de $E F$ y
48/356 (13.4\%) de sin EF. De los EF 3/18(16.6\%) no consumía ninguna fruta o vegetal y el 39\% (7/18) refirió ingesta excesiva de lácteos. 
Referente al entrenamiento esfinteriano: abandonó el uso de pañales e inició el uso del sanitario antes de los 24 meses, 194/356 (54,5\%) de los niños con EF y $10 / 18(55,5 \%)$ niños sin $\mathrm{EF}$, siendo similar la distribución entre ambos grupos.
Se refirió ausencia de escolarización o nivel primario sin adquisición de lectoescritura en 44/ 374 (11,7\%) encuestados. En la población de niños con EF ningún cuidador tuvo educación terciaria. (Tabla 4)

Tabla 4. Nivel educativo de los cuidadores de la población con y sin estreñimiento funcional N=374

\begin{tabular}{lll}
\hline Nivel educativo & $\begin{array}{l}\text { Con EF* } \\
\mathbf{n = 1 8}\end{array}$ & $\begin{array}{l}\text { Sin EF } \\
\mathbf{n = 3 5 6}\end{array}$ \\
\hline Primaria incompleta o sin escolarización & $2(11 \%)$ & $42(12 \%)$ \\
Primaria completa & $6(33 \%)$ & $67(19 \%)$ \\
Secundaria incompleta & $3(17 \%)$ & $54(15 \%)$ \\
Secundaria completa & $7(39 \%)$ & $127(35 \%)$ \\
Terciaria & 0 & $66(19 \%)$ \\
\hline$E F=$ Estreñimiento funcional & &
\end{tabular}

Los entrevistados interpretaron el estreñimiento en orden de frecuencia como: el esfuerzo al defecar 101/374 (27\%), ausencia de defecación diaria 74/374 $(19,8 \%)$, deposiciones duras 69/374 $(18,4 \%)$, deposiciones dolorosas 60/374 (16\%), necesidad de ingerir laxantes $57 / 374(15,2 \%)$ y otras $44 / 374$ $(11,8 \%) .18$ de los entrevistados consideraron más de una característica.

Respecto al conocimiento de los encuestados sobre los hábitos intestinales de sus niños, 7/374 (1,9\%) manifestaron desconocimiento sobre la frecuencia evacuatoria de estos. En 18/374 (4,8\%) casos se refirió evacuaciones solo una vez por semana y 349/374 $(93,3 \%)$ más de 2 veces por semana. En cuanto a las características de las deposiciones: se describió heces caprinas en $14 / 18$ (77,7\%); en $7 / 18$ (38,8\%) dificultosas; voluminosas y en escíbalos en $3 / 18$ $(16,6 \%)$ en niños con EF. Los niños sin EF, presentaron estas alteraciones en forma ocasional.

Fueron manejados ya con sea laxantes, supositorios o enemas por orden de frecuencia, $16 / 18(88,9 \%)$ de los niños con EF y 2/18 (11,1\%) no recibieron medicación debido a no percibirse necesario. De los niños sin EF, 119/ 356 (33,4 \%) presentaron episodios ocasionales de estreñimiento y de estos 108/119 $(88,2 \%)$ también recibieron supositorios, laxantes y enemas. En ambos grupos algunos niños tuvieron más de un tipo de intervención. (Tabla 5)

Tabla 5. Medicación administrada a los niños. n=374.

\begin{tabular}{llll}
\hline Tratamiento & $\begin{array}{l}\text { Con EF* } \\
\mathbf{n = 1 8}\end{array}$ & $\begin{array}{l}\text { Sin EF } \\
\mathbf{n}=\mathbf{3 5 6}\end{array}$ & Valor p \\
\hline Osmóticos & $10(55,6 \%)$ & $36(10,1 \%)$ & $<0,001$ \\
Supositorio & $8(44,4 \%)$ & $42(11,8 \%)$ & $<0,001$ \\
Enema & $4(22,2 \%)$ & $10(2,8 \%)$ & $<0,001$ \\
Catárticos & $1(5,6 \%)$ & $12(3,4 \%)$ & 0,622 \\
Ninguno & $2(11,1 \%)$ & $248(69,7 \%)$ & $<0,001$ \\
\hline
\end{tabular}

$E F=$ Estreñimiento funcional 


\section{DISCUSIÓN}

El estreñimiento constituye un problema de relevancia a nivel global debido a su impacto en la calidad de vida de los afectados, por lo cual un mejor conocimiento y comprensión de la realidad local pudiera favorecer un abordaje más apropiado.

Su prevalencia a nivel mundial, tanto en países en desarrollo como desarrollados, oscila en un amplio rango entre $0,7-29 \%$, con una media del $12 \%$. Hay dificultad en establecer cifras globales reales por la gran heterogeneidad de las definiciones usadas en los diferentes estudios y países ${ }^{(9)}$. Existe variación con la edad, teniendo un pico de incidencia entre los 2 y los 4 años, reportándose una prevalencia de $2.9 \%$ al año, $10.1 \%$ a los dos años y de $34 \%$ hacia la edad de escolarización $^{(2)}$ y tres periodos críticos en su aparición: la introducción de la alimentación complementaria, el entrenamiento esfinteriano, y el inicio de la escolarización ${ }^{(2,4)}$.

En el presente estudio, sobre una población pediátrica de una consulta ambulatoria, la prevalencia de $4,8 \%$ de EF, aunque dentro del margen descrito, se situó en los rangos inferiores, considerando la media reportada ${ }^{(4)}$; sin diferencias llamativas entre preescolares y escolares (4,4 y 5,1\% respectivamente). La mayor parte de la población, aunque procedente de la capital y del departamento central del país, consideradas áreas urbanas, provino de una consulta ambulatoria de un hospital público, pudiendo tener estilos de vida, prácticas alimentarias y de cuidado infantil que afecten la prevalencia del EF.

Benninga et al. ${ }^{(8)}$ citan como factor de riesgo para estreñimiento el hecho de quedar al cuidado de terceros; sin embargo, esto no pudo ser objetivado en nuestra población, pues la mayor parte de niños, tanto con EF como sin EF estaban al cuidado de sus padres.

El dolora la evacuación, citado en la literatura como principal detonador de la retención fecal y factor clave para la instalación del círculo vicioso del EF, fue referido desde el inicio del cuadro en dos de cada tres de los niños con EF en nuestra investigación, pudiendo suponerse que su presencia hubiese podido contribuir a la instalación del mismo ${ }^{(2)}$.
Se ha señalado propensión familiar, sobre todo de madres de niños con estreñimiento a presentar el problema $^{(8)}$. En coincidencia con ello y a pesar del reducido número de EF en este estudio, se observó coexistencia de historia familiar positiva en dos de cada tres niños EF, especialmente en la madre.

Con relación al rol de los factores dietéticos en el estreñimiento, en una revisión sistemática reciente. sobre el uso de fibra en niños con EF y síndrome de intestino irritable, la mayoría de estudios demostraron su beneficio, aunque hubo heterogeneidad en el diseño de estudio, duración del tratamiento, cantidad y tipo de fibra ${ }^{(12)}$. En nuestra investigación, en $4 / 10$ niños con EF se detectó ingesta de lácteos superior a la recomendada para la edad y en algunos casos ingesta nula de frutas y vegetales. En el resto de la población, la mayoría tenía ingesta de fibra dietaria inferior a las recomendaciones. Llamativamente esto no se acompañó de elevada prevalencia de EF, avalando su complejidad fisiopatológica, que requeriría la conjunción de varios factores para su instalación.

Se ha reportado que un $40 \%$ de estreñimiento en niños debuta a los 2-3 años, pudiendo favorecerlo el apresurar el control esfinteriano. Aunque más de la mitad de los entrevistados refirieron retirada de pañal y entrenamiento esfinteriano antes de los 24 meses, considerado prematuro de acuerdo a las pautas del desarrollo psicomotor del niño ${ }^{(5)}$, esto no coincidió con un aumento de la frecuencia de EF, reforzando la visión de la complejidad del problema ${ }^{(1)}$.

Las encuestas hicieron notoria la necesidad de educación sobre el concepto de EF, pues los entrevistados tenían una interpretación variable del mismo, aunque conocían los hábitos intestinales y las características de las evacuaciones de los niños. La mayoría lo asociaba con la frecuencia, más que con las características de las heces o sus síntomas. Llamó la atención la utilización de supositorios y enemas en casi todos los niños con EF, y sobre todo su uso en los casos de estreñimiento ocasional, hecho que amerita un análisis, pues, se recomienda limitarlos a casos que no respondan a medicación oral, por ser invasivas y traumáticas emocional y físicamente ${ }^{(1)}$.

El nivel educativo de los padres y cuidadores es señalado como relevante para la salud del niño y 
para la construcción de hábitos ${ }^{(1)}$. La ausencia de escolarización en esta población superó el 10\%, y el $100 \%$ de los cuidadores de niños con EF no superó el nivel secundario. Aunque es un dato importante se debe interpretar con precaución por el tamaño de la muestra y porque el contexto sociocultural no fue evaluado.

Las concepciones culturales pudieran afectar las creencias, interpretación o significado que se otorgan a los signos y síntomas sobre el hábito intestinal de niños, y propiciar prácticas de alimentación o de entrenamiento esfinteriano que influencien en el estreñimiento, derivando en un rango de variabilidad tan amplio de prevalencias reportadas. La relativa baja frecuencia de EF observada en nuestra población de estudio con relación a la media mundial reportada, pudiera atribuirse a diferencias culturales y a un manejo más natural de la construcción de los hábitos intestinales, más que a los hábitos alimentarios, pues no se ha podido objetivar ingesta adecuada de fibra.

A fin de propiciar la consulta oportuna y de evitar abordajes y prácticas desaconsejadas, se requiere mejorar el concepto de estreñimiento en los cuidadores, asesorar sobre hábitos de alimentación y entrenamiento esfinteriano, así como promover la educación médica continua, socializando los Criterios de Roma IV para el diagnóstico y tratamientos oportunos del $\mathrm{EF}^{(7)}$.

Para clarificar la relación del antecedente familiar positivo, el nivel educativo de los cuidadores, la construcción de los hábitos intestinales y de alimentación con el EF se requiere proyectar estudios para dicho propósito.
Cabe mencionar como una limitación del estudio, además del tamaño muestral que considere el impacto de niveles socioculturales y estilos de vida para aclarar la diferencia de prevalencia y las repercusiones del problema en la salud de la infancia, la falta de un test de fiabilidad de la encuesta. Sin embargo, aporta datos importantes sobre el EF, que podrían ser verificados con estudios más amplios y sobre la base de cuestionarios validados, fiables en el futuro.

\section{CONCLUSIONES}

La prevalencia de estreñimiento fue inferior al promedio mundial reportado.

El dolor a la evacuación se refirió en dos tercios de los niños con EF. Se observó coincidencia con estreñimiento materno. Los hábitos de alimentación o de entrenamiento esfinteriano tanto en EF o sin EF no se ajustaron a las recomendaciones para la edad. El nivel educativo de la población fue bajo. Por el tamaño de la muestra no se pudo clarificar el impacto real de estos aspectos en nuestra población. La interpretación del concepto de estreñimiento fue variada, asociándolo esencialmente a la frecuencia, sin considerar las características como volumen o consistencia de las deposiciones y los síntomas asociados. Fueron utilizados supositorios y enemas tanto en EF como en estreñidos ocasionales.

Considerando que la mayoría de factores asociados al EF infantil son modificables, una atención a los aspectos dietéticos y al aprendizaje de hábitos es crucial, para lo cual habría que involucrar a padres, niños, pediatras y educadores. 


\section{REFERENCIAS}

1. Remes-Troche JM, Coss-Adame E, Lopéz-Colombo A, Amieva-Balmori M, Carmona Sánchez R, Charúa Guindic $\mathrm{L}$, et al. Consenso mexicano sobre estreñimiento crónico. Revista de Gastroenterología de México. 2018, 83(2):168189. doi: http://dx.doi.org/10.1016/j.rgmx.2017.12.005

2. Avelar Rodríguez D, Toro-Monjaraz EM, RamírezMayans JA. Constipacion funcional en pediatria: Criterios de Roma IV, diagnóstico y tratamiento. Acta Pediatr Mex. 2018;39(1):81-84.

3. Oswari H, Alatas FS, Hegar B, Cheng W, Pramadyani A, Benninga MA, et al. Epidemiology of Paediatric constipation in Indonesia and its association with exposure to stressful life events. BMC Gastroenterology. 2018; 18(146).doi: https://doi.org/10.1186/s12876-018-0873-0

4. Mata M, da Cuña R. Estreñimiento y encopresis. Pediatr Integral. 2015; 19:127-38.

5. Howarth LJ, Sullivan PB. Management of chronic constipation in children. Pediatrics and Child Health. 201; 26(10):415-422. doi: https://dx.doi.org/10.1016/j.paed. 2016.06.007

6. Devanarayana NM, Rajindrajith S. Association between constipation and stressful life events in a cohort of Sri Lankan children and adolescents. J Trop Pediatr. 2010;56(3):144-8. doi: https://dx.doi.org/10.1093/tropej/fmp077
7. Benninga MA, Faure C, Hyman PE, St James Roberts I, Schechter NL, Nurko S. Childhood Functional Gastrointestinal Disorders: Neonate/Toddler. Gastroenterology. 2016; 15:S0016-5085(16)00182-7. doi: https://dx.doi.org/10.1053/j.gastro.2016.02.016

8. Blesa Baviera LC. Trastornos digestivos funcionales pediátricos. Criterios Roma IV. En: AEPap (ed.). Curso de Actualización Pediatría, Madrid: Lúa Ediciones;2017:99-114.

9. Vandenplas Y, Benninga M, Broekaert I, Falconer J, Gottrand F, Guarino F, et al. Functional gastro-intestinal disorder algorithms focus on early recognition, parental reassurance and nutritional strategies. Acta Paediatr. 2016;105(3):244-52. doi: https://dx.doi.org/10.1111/apa.13270

10.Ministerio de Salud. Guía De Alimentación Del Niño(A) Menor De 2 Años. Guía De Alimentación Hasta La Adolescencia. 4ed. Chile; 2015.

11. García Pérez MA, Martínez Granero MA. Desarrollo psicomotor y signos de alarma. En: AEPap (ed.). Curso de Actualización Pediatría, Madrid: Lúa Ediciones;2016:81-93.

12. Axelrod CH, Saps M. The Role of Fiber in the Treatment of Functional Gastrointestinal Disorders in Children. Nutrients. 20183;10(11):1650. doi: https://dx.doi.org/10. 3390/nu10111650 